\title{
Electron-nuclear double resonance
}

\author{
Leonid Kulik · Wolfgang Lubitz
}

Received: 4 December 2008/Accepted: 31 December 2008/Published online: 31 January 2009

(C) Springer Science+Business Media B.V. 2009

\begin{abstract}
The application of electron-nuclear double resonance (ENDOR) spectroscopy for the investigation of photosynthetic systems is reviewed. The basic principles of continuous wave and pulse ENDOR are presented. Selected examples of the application of the ENDOR technique for studying stable and transient paramagnetic species, including cofactor radical ions, radical pairs, triplet states, and the oxygen-evolving complex in plant Photosystem II (PSII) are discussed. Limitations and perspectives of ENDOR spectroscopy are outlined.
\end{abstract}

Keywords Continuous wave ENDOR - Pulse ENDOR . Photosynthesis · Primary donor radical cation .

Quinone acceptor radical anion - Carotenoid triplet state . Radical pair - Manganese cluster .

Oxygen-evolving complex · Photosystem II .

Photosystem I

$\begin{array}{ll}\text { Abbreviations } \\ \text { BChl } & \text { Bacteriochlorophyll } \\ \text { Car } & \text { Carotenoid } \\ \text { CW } & \text { Continuous wave } \\ \text { EPR } & \text { Electron paramagnetic resonance } \\ \text { ENDOR } & \text { Electron nuclear double resonance } \\ \text { ESE } & \text { Electron spin echo } \\ \text { ESEEM } & \text { Electron spin echo envelope modulation } \\ \text { HFI } & \text { Hyperfine interaction }\end{array}$

L. Kulik

Institute of Chemical Kinetics and Combustion, Institutskaya 3, 630090 Novosibirsk, Russia

W. Lubitz ( $₫)$

Max-Planck-Institut für Bioanorganische Chemie,

Stiftstrasse 34-36, 45470 Mülheim an der Ruhr, Germany

e-mail: lubitz@mpi-muelheim.mpg.de

$\begin{array}{ll}\text { mw } & \text { Microwave frequency } \\ \text { NMR } & \text { Nuclear magnetic resonance } \\ \text { NQI } & \text { Nuclear quadrupole interaction } \\ \text { rf } & \text { Radio frequency } \\ \text { OEC } & \text { Oxygen-evolving complex } \\ \mathrm{P} & \text { Primary donor } \\ Q_{A, B} & \text { Quinone acceptors } A, B \\ \mathrm{RC} & \text { Reaction center } \\ \mathrm{RP} & \text { Radical pair } \\ \text { TRIPLE } & \text { Electron nuclear nuclear triple resonance } \\ \text { ZFS } & \text { Zero field splitting }\end{array}$

\section{Introduction}

Electron-nuclear double resonance (ENDOR) has been introduced by Feher (1956) in solid state physics and later extended to radicals in solution by Hyde and Maki (1964). The technique has been extensively used in photosynthesis research (reviewed in Möbius et al. 1989, Lubitz and Lendzian 1996, Rigby et al. 2001, Britt et al. 2004). ENDOR combines electron paramagnetic resonance (EPR) and nuclear magnetic resonance (NMR) spectroscopy, but their roles are different. The EPR signal is measured at a fixed magnetic field, and its intensity is varied by the applied scanned radio frequency (rf) irradiation (NMR). ENDOR is sensitive only to paramagnetic species. Fortunately, such species frequently occur in photosynthesis. Many photosynthetic reactions involve radicals, radical pairs (RPs), and triplet states and active centers of the proteins and enzymes often contain transition metal ions. Thus, ENDOR is able to probe the most interesting parts of the photosynthetic machinery.

The additional NMR dimension increases the resolution of ENDOR as compared to EPR and makes it the method 
of choice for the investigation of complex paramagnetic systems with a large number of magnetic nuclei. ENDOR spectroscopy is primarily directed to study the magnetic interactions of the unpaired electron spin with the spins of magnetic nuclei (hyperfine interaction, HFI). These nuclei can belong either to the molecule on which the unpaired electron is localized, or to the surrounding molecules. In favorable cases, the nuclear quadrupole interaction (NQI) experienced by nuclei with spin $I>1 / 2$ can be tested by ENDOR. The strength of the HFI and the NQI is intimately related to the electron spin and charge density distribution of the molecule, respectively. Therefore, their detection offers a deep insight into the electronic structure of the studied systems, which is crucial for understanding their chemical reactivity and function.

The two main branches of ENDOR, continuous wave (CW) and pulse, are based on $\mathrm{CW}$ and pulse EPR, respectively. Pulse ENDOR requires the detection of the electron spin echo (ESE) signal, which limits its application to systems with a sufficiently large transverse electron spin relaxation time $\left(T_{2}>100 \mathrm{~ns}\right)$. This makes pulse ENDOR not suitable for studies of liquid samples and generally requires low-temperature experiments. CW ENDOR is free from this limitation and allows the experiments to be performed under physiological conditions. However, the technique requires "fine tuning" of the longitudinal relaxation times of the electron and nuclear spins for optimum signal intensities. Due to the strong temperature dependence of these relaxation rates, pulse ENDOR is usually superior to CW ENDOR at low temperatures.

This article starts with a brief theoretical section, where the most important equations are presented. Then selected examples of ENDOR studies of photosynthetic systems are reviewed. Furthermore, limitations and perspectives of the technique are discussed.

\section{Theory}

Spin system

The simplest system for which ENDOR can be used is a radical with the electron spin $S=1 / 2$ which has one nucleus with nuclear spin $I=1 / 2$. First, we assume that hyperfine coupling between them is isotropic. If the $g$ tensor is also isotropic, the spin-hamiltonian $H$ of this system is (in frequency units):

$\frac{H}{h}=\frac{g \beta_{\mathrm{e}}}{h} B_{0} S_{\mathrm{z}}-\frac{g_{\mathrm{n}} \beta_{\mathrm{n}}}{h} B_{0} I_{\mathrm{z}}+a(S I)$.

The first term in this equation describes the electron Zeeman interaction, the second term describes the nuclear Zeeman interaction, and the third describes the HFI. Here, $h$ is Planck's constant, $\beta_{\mathrm{e}}$ is the Bohr magneton, $g$ is the electronic $g$-value, $\beta_{\mathrm{n}}$ is the nuclear magneton, $g_{\mathrm{n}}$ is the nuclear $g$-value, $a$ is the HFI constant, $S$ and $I$ are the operators of the electron and nuclear spin. We assumed that the constant magnetic field of the EPR spectrometer $B_{0}$ is directed along the $z$-axis of the laboratory frame. The spin-hamiltonian in Eq. 1 is typical for a radical in liquid solution, for which fast rotation averages out all anisotropic interactions.

In the strong field approximation, the first term is dominant in Eq. 1. Thus, all energy levels of the system are characterized by definite $z$-projections of the electron and nuclear spin, $m_{S}= \pm 1 / 2$ and $m_{I}= \pm 1 / 2$, respectively. The first-order eigenvalues are then:

$E\left(m_{S}, m_{I}\right) / h=v_{\mathrm{e}} m_{S}-v_{\mathrm{n}} m_{I}+a m_{S} m_{I}$,

where $v_{\mathrm{e}}=g \beta_{\mathrm{e}} B_{0} / h$ is the electron frequency and $v_{\mathrm{n}}=$ $g_{\mathrm{n}} \beta_{\mathrm{n}} B_{0} / h$ is the nuclear Larmor frequency. The respective energy level diagram is shown in Fig. 1.

In the EPR experiment, the selection rules $\Delta m_{S}= \pm 1$ and $\Delta m_{I}=0$ hold. Therefore, two allowed EPR transitions exist in the described system. In an ENDOR experiment, the rf field drives also the NMR transitions with the selection rules $\Delta m_{S}=0$ and $\Delta m_{I}= \pm 1$. The frequencies of these transitions are:

$v_{\mathrm{ENDOR}}^{ \pm}=\left|v_{\mathrm{n}} \pm a / 2\right|$.

\section{Continuous wave ENDOR}

The ENDOR effect appears when both microwave (mw) and rf fields are in resonance with the EPR and NMR transitions, respectively, and these transitions have a common energy level. For a stable radical in thermodynamic equilibrium,

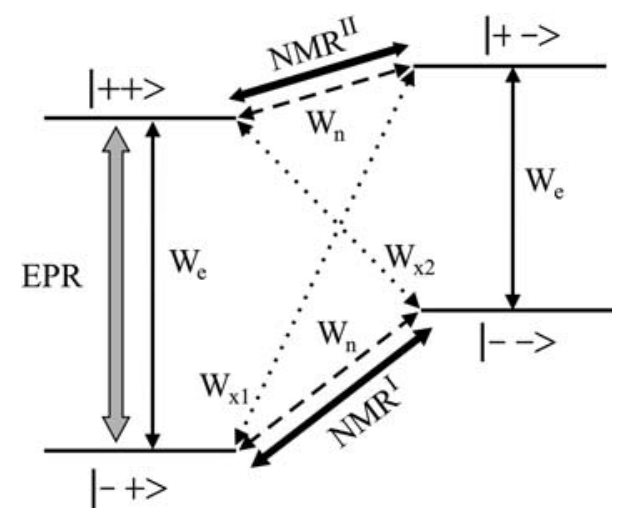

Fig. 1 Energy level diagram for the coupling of one electron spin $(S=1 / 2)$ with one nuclear spin $(I=1 / 2)$. The spin functions are indicated on the four resulting levels; EPR and NMR transitions are indicated together with the electron spin $\left(W_{\mathrm{e}}\right)$, nuclear spin $\left(W_{\mathrm{n}}\right)$ and cross-relaxation rates $\left(W_{x 1}, W_{x 2}\right)$. In a CW ENDOR experiment, the NMR resonances (black arrows) are detected via the change of a simultaneously irradiated saturated EPR line (gray arrow); for further details, see text and (Kurreck et al. 1988) 
CW ENDOR can be described as NMR-induced partial desaturation of a saturated EPR line.

The various spin relaxation processes for the $S=1 / 2$, $I=1 / 2$ system are shown as dashed lines in Fig. 1. The rate of longitudinal spin relaxation (population relaxation) of the electron spin is $W_{\mathrm{e}}$, that of the nuclear spin is $W_{\mathrm{n}}$, and the rates of the electron-nuclear cross-relaxation are $W_{x 1}$ and $W_{x 2}$.

In CW ENDOR, one EPR transition is saturated by mw irradiation, as indicated by the thick vertical arrow in Fig. 1. Simultaneously, one NMR transition $\left(\mathrm{NMR}^{\mathrm{II}}\right.$ or $\mathrm{NMR}^{\mathrm{I}}$ ) is saturated by the $\mathrm{rf}$ field. This opens an alternative relaxation path for the pumped electron spin. For the case of $\mathrm{NMR}^{\mathrm{II}}$ pumping, it can relax via a two-step pathway $W_{\mathrm{e}}(|+-\rangle \leftrightarrow|--\rangle), \quad W_{\mathrm{n}}(|--\rangle \leftrightarrow|-+\rangle) \quad$ or directly by $W_{x 1}(|+-\rangle \leftrightarrow|-+\rangle)$. The extent to which the additional relaxation bypass desaturates the EPR line determines the intensity of the ENDOR signal. Thus, the ENDOR line intensity usually does not reflect the number of contributing nuclei, in contrast to NMR or EPR. In the limit of strong NMR saturation and with the simplifying assumption $W_{x 1}=W_{x 2}=0$, the intensity of the ENDOR signal $E$ is:

$E=\frac{1}{2\left(2+b+b^{-1}\right)}$

where $b=W_{\mathrm{n}} / W_{\mathrm{e}}$. The quantity $E$ is usually called "ENDOR enhancement" and is measured as the relative change of the EPR signal.

It is obvious that $E$ strongly depends on the relaxation properties of the system (Plato et al. 1981). One needs to carefully optimize the respective rates, e.g., by variation of temperature, to reach the "matching condition" $W_{\mathrm{n}}=W_{\mathrm{e}}$, which corresponds to the maximum ENDOR enhancement $E_{\max }=1 / 8$. Cross-relaxation might increase this value. However, since usually $W_{x 1} \neq W_{x 2}$ holds, the asymmetric relaxation network produces an asymmetry of the ENDOR spectrum.

For more complicated systems with $k>1$ nuclei and with $I=1 / 2$, the situation is qualitatively similar. For this case Eq. 1 can be easily generalized to:

$\frac{H}{h}=v_{\mathrm{e}} S_{z}-\sum_{i} v_{\mathrm{n}(i)} I_{z(i)}+\sum_{i} a_{i}\left(S I_{i}\right)$

where the index $i$ runs over all nuclei. If these nuclei are non-equivalent the system has $2^{k}$ EPR transitions and only $2 k$ ENDOR transitions with the frequencies:

$v_{\mathrm{ENDOR}}=\left|v_{\mathrm{n}(i)} \pm a_{i} / 2\right|$.

This illustrates the power of ENDOR spectroscopy for simplification of the spectra as compared to EPR. Although ENDOR is less sensitive than EPR, it is many orders of magnitude more sensitive than NMR experiments on paramagnetic systems, which is due to the enormous increase in the linewidth as compared to NMR on diamagnetic molecules.

\section{Special TRIPLE}

As can be seen from Fig. 1, simultaneous pumping of both NMR transitions increases the effect of the relaxation bypass. It is especially pronounced when $W_{\mathrm{n}}, W_{x 1}$, $W_{x 2} \ll W_{\mathrm{e}}$. This is used in "Special TRIPLE" experiment, in which the sample is irradiated with two rf frequencies $v_{1}=v_{\mathrm{n}}-v_{\mathrm{T}}, v_{2}=v_{\mathrm{n}}+v_{\mathrm{T}}$, with $v_{\mathrm{T}}$ scanned (Freed 1969; Dinse et al. 1974). In such experiment, the line intensities are approximately proportional to the number of nuclei contributing to this line.

\section{General TRIPLE}

General TRIPLE can be applied to systems consisting of one electron spin and several nuclear spins (Biehl et al. 1975). We will consider the simplest case: one electron with $S=1 / 2$ coupled to two nuclei with $I_{1}=I_{2}=1 / 2$. The system has four nuclear spin transitions, and each of them is doubly degenerate. In General TRIPLE, similar to the ENDOR experiment, the rf frequency $v_{1}$ is scanned. It is different from ENDOR, in that one of the nuclear spin transitions is additionally pumped by a fixed frequency $v_{2}$. This saturation of one ENDOR line affects the intensities of all other lines, because additional relaxation pathways become active. The most important feature of General TRIPLE is that the changes in the observed line intensity, relative to ENDOR, depend on the relative signs of the HFI constants $a_{1}$ and $a_{2}$. The analysis, carried out for the case of negligible cross-relaxation, and small HFI constants $\left|a_{1} / 2\right|<v_{\mathrm{n}(1)},\left|a_{2} / 2\right|<v_{\mathrm{n}(2)}$, shows that if the high-frequency line of the second nuclear spin is pumped, the intensity of the high-frequency line of the first nuclear spin decreases for the case of the same signs of $a_{1}$ and $a_{2}$. Additionally, the intensity of the high-frequency line of the first nuclear spin increases. This intensity pattern is inverted for the case of opposite signs of $a_{1}$ and $a_{2}$. Note that the distribution is also reversed in heteronuclear General TRIPLE experiments if the two nuclei have different signs of the magnetic momentum (e.g., for ${ }^{1} \mathrm{H}$ and ${ }^{15} \mathrm{~N}$ ).

\section{Pulse ENDOR}

Most of the pulse ENDOR techniques are based on the ESE effect. The echo signal is created by the proper mw pulse sequence. The rf pulse, applied during the "mixing period" of the pulse sequence, drives nuclear spin transitions, thus changing the ESE intensity. The pulse ENDOR signal is measured as the amplitude of this change when the rf frequency is scanned. 
There are two most popular pulse ENDOR sequences: Davies and Mims ENDOR (Davies 1974; Mims 1965). The principle of pulse ENDOR can be best understood for the $S=1 / 2, I=1 / 2$ system. In Davies ENDOR (Fig. 2), an mw inversion-recovery pulse sequence $(\pi-\mathrm{T}-\pi / 2-\tau-\pi-\tau-$ echo) is used. First, one EPR transition is inverted by the $\pi$ pulse, the so-called preparation pulse. In order to avoid the inversion of the second EPR transition, the amplitude of the mw field $B_{1}$ should be properly adjusted ( $B_{1} \leq a$ should hold). Therefore, Davies ENDOR is useful for systems with large HFIs. For the case of a stable radical in thermal equilibrium, the initial polarization of the EPR transition is positive. The mw $\pi$-pulse inverts this polarization. During the $T$ interval, the rf pulse changes the population of the nuclear sublevels, and thereby the polarization of the EPR transition is partially restored. This effect is detected by the echo intensity, i.e., by the final part of the pulse sequence $\pi / 2-\tau-\pi-\tau-$ echo.

In Mims ENDOR, both EPR transitions are excited by the applied stimulated echo mw pulse sequence $(\pi / 2-\tau-\pi /$ $2-\mathrm{T}-\pi / 2-\tau-\mathrm{echo})$. This limits the application of this method to relatively small HFI constants $\left(B_{1} \geq a\right)$. A spin level population diagram is not adequate for the description of Mims ENDOR, because the transverse components of the electron spin magnetization (coherencies) are involved here. Qualitatively, Mims ENDOR can be explained as a partial defocusing of the ESE. The rf $\pi$-pulse changes $m_{I}$, which in turn changes the frequency of the electron spin Larmor precession. Thus, the frequency of this precession during the first and the second $\tau$ period differs by the value of $a$. At the moment of the echo formation, the precessing magnetization acquires the additional phase $\Delta \phi=a \tau$, so the echo intensity is proportional to

$S_{y}=\cos (a \tau)$.

As evident from Eq. 7, no ENDOR effect is observed when $a \tau=2 \pi n$, where $n$ is an integer number. Therefore, for the

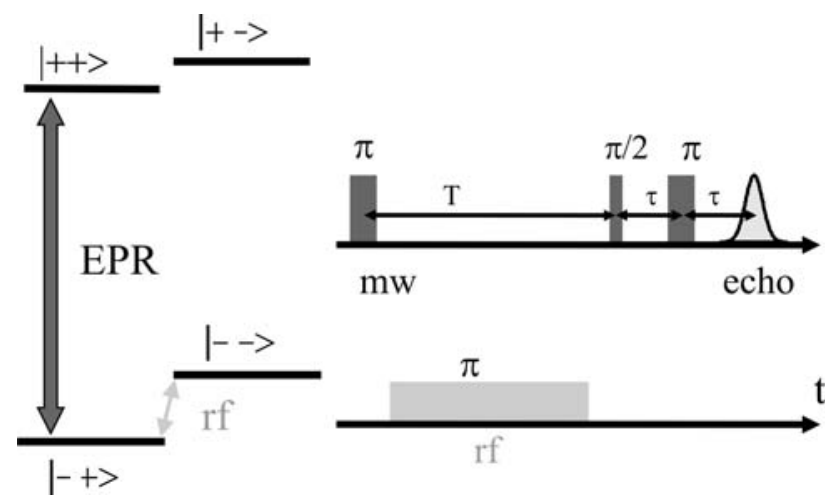

Fig. 2 Energy level diagram (left) for an $S=I=1 / 2$ system and pulse scheme (right) for the Davies ENDOR experiment (Davies 1974; Schweiger and Jeschke 2001) given $\tau$ value "blindspots," or regions with severely decreased ENDOR sensitivity appear in the Mims ENDOR spectrum around $a=2 \pi n / \tau$. The presence of such blindspots is a major drawback of Mims ENDOR spectroscopy.

If the strength of the HFI is comparable or larger than the nuclear Larmor frequency, the hyperfine enhancement effect manifests itself both in CW and pulse ENDOR. It is caused by the influence of the rf field on the electron spin. Due to this influence, the effective rf field experienced by the nuclear spins becomes dependent on $m_{S}$ and on the HFI strength, which leads to a change of the ENDOR line intensity. A detailed description of this and several other features of ENDOR can be found in (Schweiger and Jeschke 2001).

\section{Experimental}

The setup for ENDOR experiments is based on that for CW or pulse EPR. The difference is that for ENDOR, an $\mathrm{rf}$ source and amplifier is necessary. The rf output from this amplifier is fed into the rf coils, placed at the EPR cavity. The geometry of these coils is typically chosen in such way that the magnetic component of the rf field $B_{2}$ is perpendicular to both $B_{0}$ and $B_{1}$. For the description of ENDOR instrumentation refer to (Kevan and Kispert 1976; Kurreck et al. 1988, Poole 1983).

\section{Examples of application}

The radical cation of $\mathrm{BChl} a$ in liquid solution

Knowledge of the electronic structure of the radical ions of $\mathrm{BChl} a$ is important for understanding the respective radicals occurring in the primary charge separation process in bacterial photosynthetic reaction centers (RCs). The results obtained in organic solvents are needed to trace the changes that occur when these species are bound to the RC protein.

Here the radical cation of $\mathrm{BChl} a$ is described as a model for the primary donor $P_{865}^{\bullet+}$ in the RC. The EPR spectrum of Bchl $a^{\bullet+}$, chemically generated in solution exhibits the same $g$ factor but the Gaussian line is about 1.4 times broader than that of $P_{865}^{\bullet+}$. This was interpreted as resulting from the formation of a BChl-dimer in the RC. The HFI constants are larger for $\mathrm{BChl} a^{\bullet+}$, but they still can be resolved only in ENDOR or TRIPLE experiments (Lubitz et al. 1997).

The EPR/ENDOR/TRIPLE results are shown and described in Fig. 3. A simplification of the ENDOR spectrum and a partial assignment of the HFI constants were achieved by the selective deuteration of $\mathrm{BChl} a^{\bullet+}$. It is 

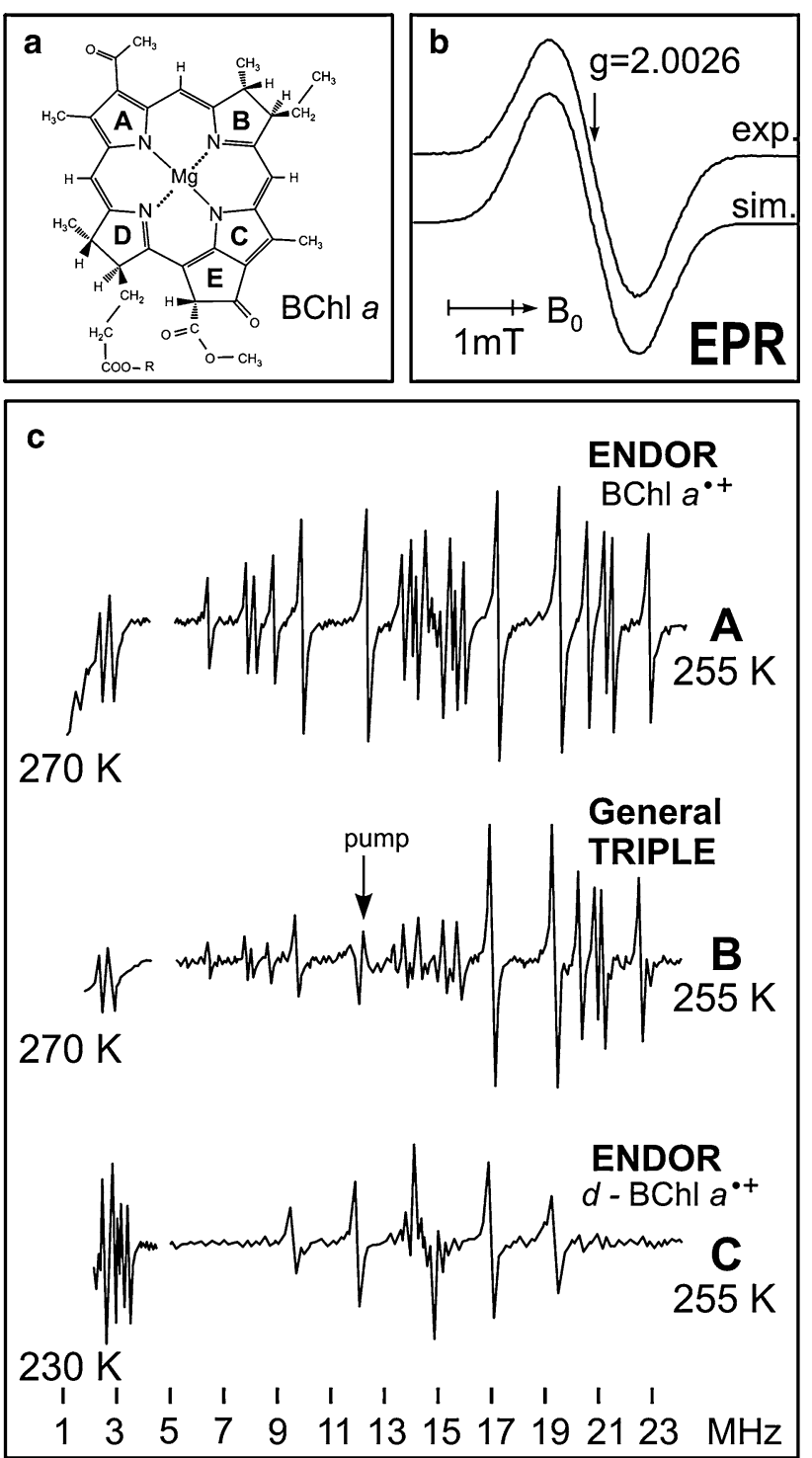

Fig. $3 \mathrm{CW}$ EPR, ENDOR, and TRIPLE resonance experiments at Xband on the bacteriochlorophyll $a$ radical cation in isotropic solution (iodine oxidation, $\mathrm{CH}_{2} \mathrm{Cl}_{2} / \mathrm{CH}_{3} \mathrm{OH}$ ) (Lubitz et al. 1997). a: Molecular structure of $\mathrm{BChl} a$. b: EPR spectrum in isotropic solution with simulation using the hyperfine couplings from ENDOR. c: ${ }^{1}{ }^{1} \mathrm{H}$ ENDOR spectrum showing 11 line pairs which yield 11 isotropic HFIs. In the low frequency range, three ${ }^{14} \mathrm{~N}$ HFI constants could be resolved (HFI constants for all four nitrogens were obtained for an ${ }^{15} \mathrm{~N}$ labeled Bchl $a^{\bullet+}$ ). B General TRIPLE experiment yielding the relative signs of all HFI couplings (including ${ }^{14} \mathrm{~N}$ ) via intensity changes relative to the pumped line pair. C ENDOR of a partially deuterated $\mathrm{Bchl} a^{\bullet+}$ that carries protons essentially only at the $\mathrm{CH}_{3}$ groups of rings $\mathrm{A}$ and $\mathrm{C}$. The respective ${ }^{2} \mathrm{H}$ ENDOR spectrum at low frequencies is also shown. For further details, see (Lubitz et al. 1997)

shown that the combination of ENDOR/TRIPLE with isotope substitution is extremely useful for studying paramagnetic systems with a large number of different magnetic nuclei. Using this approach, the authors determined the isotropic HFI values for nearly all nuclei of $\mathrm{BChl} a^{\bullet+}$, including ${ }^{14} \mathrm{~N}$ and the central ${ }^{25} \mathrm{Mg}$. These values are perfectly reproduced in quantum chemical calculations, (Sinnecker et al. 2000).

The radical cation of the primary electron donor $P_{865}^{\bullet+}$ in bacterial RCs

The primary electron donor $P_{865}$ is a part of the lightinduced electron transfer chain in bacterial RCs. According to the X-ray structure, it consists of a BChl $a$ dimer. In the photosynthetic process, upon absorption of a light quantum by $P_{865}$, this species donates an electron to a nearby acceptor, leaving behind a radical cation $P_{865}^{\bullet+}$. This can also be created artificially in the RC by chemical oxidation of $P_{865}$. The electronic structure of the primary electron donor and its radical cation is of particular interest, since this species is situated at the interface of exciton and electron transfer and is also of crucial importance for the charge recombination process.

The X-band EPR spectrum of $P_{865}^{\bullet+}$ is a broad unresolved Gaussian line, which indicates that HFI from many nuclei contribute to the EPR, while the effect of $g$-anisotropy is small. To obtain HFI values of individual nuclei, CW ENDOR and TRIPLE spectroscopies were applied to $P_{865}^{\bullet+}$ in liquid and frozen solution as well as in single crystal of bacterial RCs (Lendzian et al. 1993). About 10 lines were resolved in the ${ }^{1} \mathrm{H}$ Special TRIPLE experiment, and their angular dependence was obtained in three crystallographic planes (Fig. 4), which allowed the determination of the complete HFI tensors, including principal values and principal axes directions, for the most prominent protons.

An assignment of the HFI values was achieved by the evaluation of the axes of the anisotropic tensors while the isotropic part gave information about the spin density distribution. The data analysis, together with quantum chemical calculations (Lendzian et al. 1993), showed that the spin density is delocalized over the BChl-dimer. This distribution is asymmetric with approximately $2: 1$ weights for the L- and the M-half of the dimer. Since the two BChl $a$ molecules are chemically identical, this indicates that it is the protein environment of the RC that shifts the energies of the molecular orbitals of the bacteriochlorophylls in $P_{865}^{\bullet+}$. Thereby the redox potentials are fine-tuned (e.g., by hydrogen bonding) for optimum efficiency of the electron transfer in the RC (Lubitz et al. 2002).

The primary electron acceptor $Q_{A}^{\bullet-}$ in bacterial RCs

Although the final quinone acceptors in the bacterial RC, $Q_{A}$ and $Q_{B}$, are chemically identical, their properties in the ET chain are different. It has been shown that the EPR and ENDOR spectra of the respective radical anions, observed in Zn-substituted RCs, are also different (Lubitz and Feher 1999). 

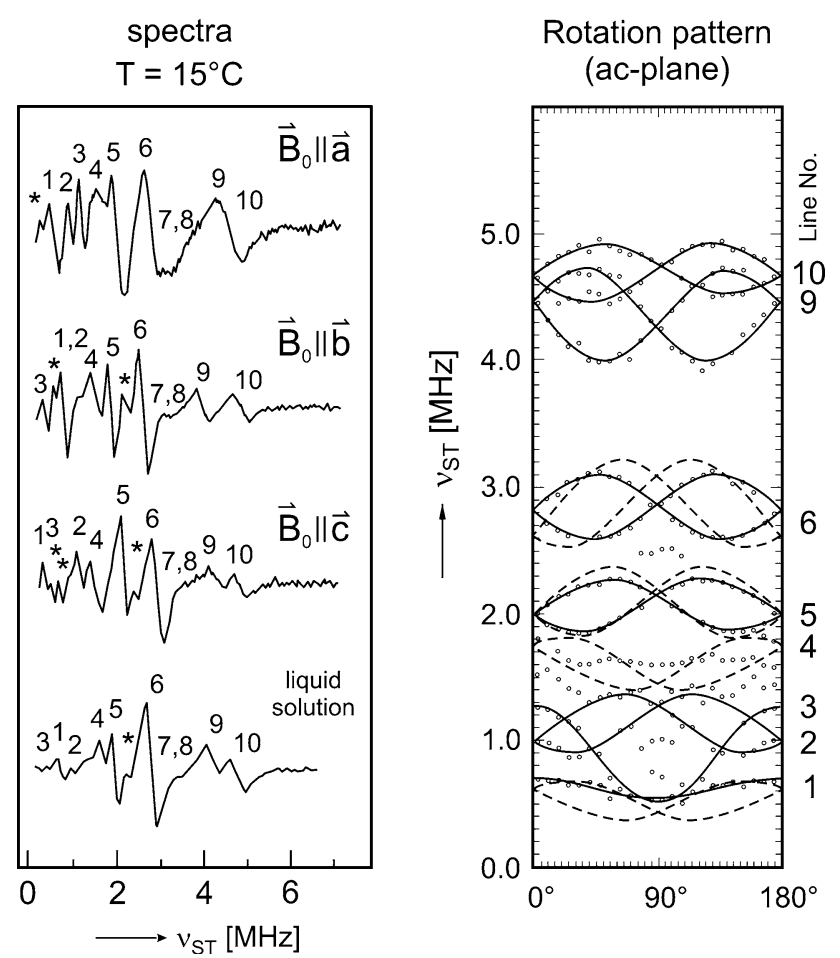

Fig. $4{ }^{1} \mathrm{H}$ Special TRIPLE spectra of the primary donor radical cation $P_{865}^{\bullet+}$ at ambient temperature in RC single crystals of Rhodobacter $(R b$.) sphaeroides $\mathrm{R}-26$, taken with the external field $B_{0}$ along the three crystallographic axes $(a, b, c)$ of the unit cell (space group $\mathrm{P} 2{ }_{1} 2_{1} 2_{1}$ ); a comparison is made with the respective spectrum in isotropic solution. On the right, the angular dependence of the line frequencies in the crystallographic $a c$-plane is shown. For details, see (Lendzian et al. 1993)

This has been traced back to a difference in the interaction with the protein surrounding. Here, we discuss the spectral features of the radical anion of $Q_{A}$. At cryogenic temperature, the electron transfer between the two quinone acceptors $Q_{A}$ and $Q_{B}$ is blocked. The same occurs if $Q_{B}$ is selectively removed. Under such conditions, $Q_{A}^{--}$is created by the illumination or chemical reduction and can be easily trapped.

It has been shown that the hydrogen bonding of $Q_{A}^{\bullet-}$ to the $\mathrm{RC}$ is of particular importance; it is probably responsible for the very unusual chemical properties of this quinone in the RC, compared with the same quinone in organic solution. The geometry of the hydrogen bonds of $Q_{A}^{--}$was probed by Q-band CW ENDOR (Flores et al. 2007). Selective deuteration opened the possibility to study separately the exchangeable (H-bonding) and non-exchangeable protons of $Q_{A}^{\bullet-}$. The increased spectral resolution at Q-band, compared with conventional X-band $(9.5 \mathrm{GHz})$, allowed obtaining ENDOR spectra at different field positions in the EPR, corresponding to particular sets of orientations of $Q_{A}^{\bullet-}$ (Fig. 5). For some $B_{0}$ values, for example, at position $\mathrm{B}_{11}$, single-crystal type ENDOR spectra were obtained. Numerical simulations of the ${ }^{1} \mathrm{H}$ and ${ }^{2} \mathrm{H}$ ENDOR spectra yielded the HFI and, for deuterons, also the NQI tensors for
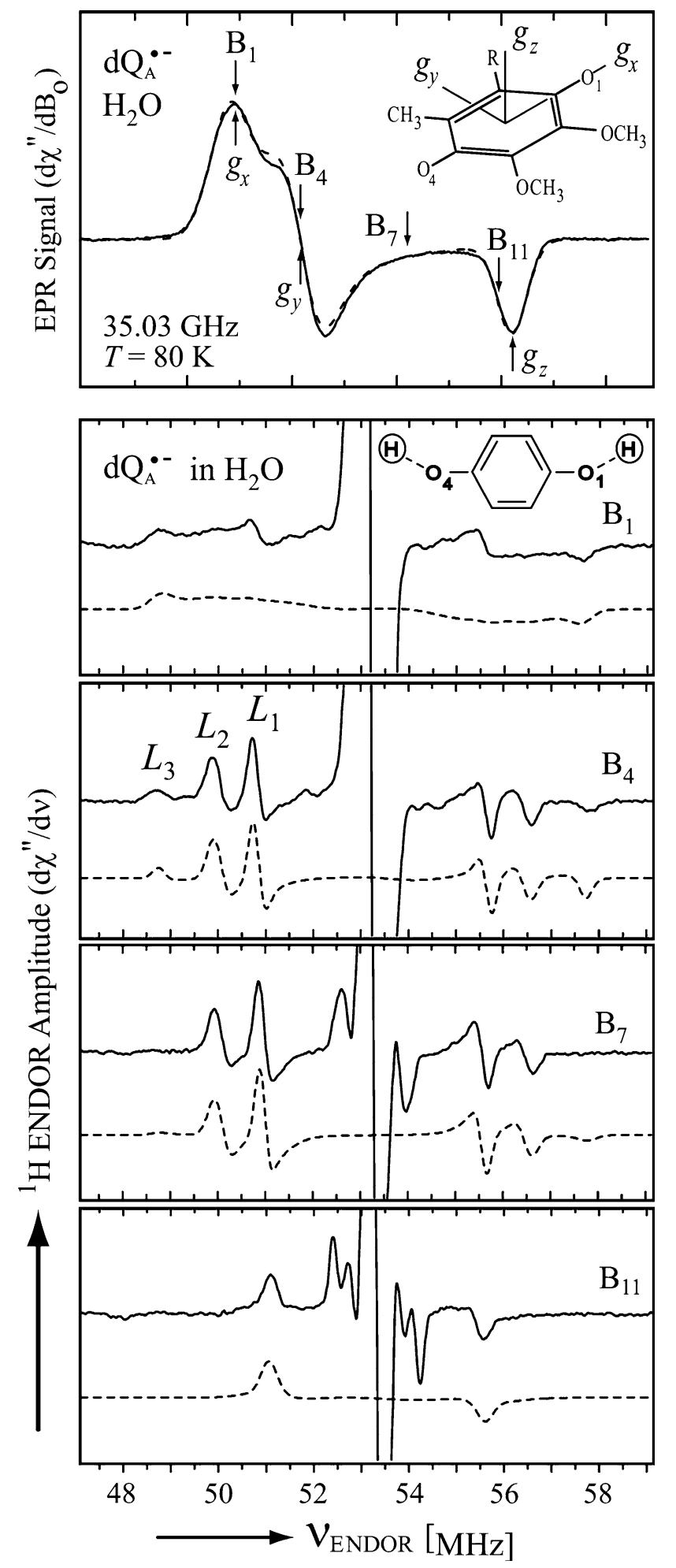

the hydrogen-bonded nuclei. Using standard relations, the hydrogen-bonding $(\mathrm{O} \ldots \mathrm{H})$ distances were determined from the main NQI tensor parameter $P_{z}$ for both carbonyl groups of $Q_{A}^{\bullet-}\left(r_{1}=1.73 \AA, r_{2}=1.60 \AA\right)$. These distances are significantly smaller (about $0.3 \AA$ ) than those for neutral $Q_{A}$, obtained from the X-ray structure of the RC, and also 
4 Fig. 5 CW EPR and ENDOR spectra at Q-band of the primary ubiquinone radical anion $Q_{A}^{\bullet-}$ in Zn-substituted RCs of Rb. sphaeroides R-26. Note that the experiments were done on fully deuterated quinone in $\mathrm{H}_{2} \mathrm{O}$ buffer. Top: EPR spectrum with simulation yielding the principal $g$-tensor components; the insert shows the quinone structure including the orientation of the g-tensor axes. Bottom: ${ }^{1} \mathrm{H}$ ENDOR spectra at four different field positions in the EPR spectrum (top) providing orientational selection with respect to the $g$-tensor axes. Note that only protons of the surrounding of the quinone radical anion are detected (matrix line, protons $\mathrm{H}$-bonded to the keto groups). The analysis, together with ${ }^{2} \mathrm{H}$ ENDOR experiments, gave information on the strength and geometry of the hydrogen bonds between protein and quinone that play a crucial role in determining the electronic structure of the primary quinone acceptor in the RC. For further details, see (Flores et al. 2007)

smaller than those obtained for typical quinone radical anions in solution. The observed asymmetry of the hydrogen bonds and their shortening upon reduction of $Q_{A}$ suggests that they play an important role in the energetic stabilization of $Q_{A}^{\bullet-}$ and the fine-tuning of the electron transfer rates in the RC (Sinnecker et al. 2006).

The oxygen-evolving complex in plant Photosystem II

The key event of oxygenic photosynthesis-light-driven oxidation of water with the release of molecular oxygenis catalyzed by the oxygen-evolving complex (OEC) of PSII. The heart of the OEC is an exchange-coupled oxygen-bridged tetranuclear manganese-calcium cluster. Because of low resolution of the present X-ray structure of PSII and the occurrence of radiation damage of the crystals, the structure of this cluster is under severe debate at present. Among the questions to be solved are the oxidation states of the individual Mn ions, their mutual positions and the exchange couplings among them. These features of the electronic structure of the cluster are crucial for understanding the mechanism of the photosynthetic water splitting process.

During the catalytic cycle (Kok cycle), the OEC passes through several distinct redox states ( $\mathrm{S}$-states, $\mathrm{S}_{0}-\mathrm{S}_{4}$ ). The $\mathrm{S}_{0}$ and $\mathrm{S}_{2}$ states have a ground state of $S=1 / 2$, and due to the coupling with the ${ }^{55} \mathrm{Mn}$ nuclei $(I=5 / 2)$ produce multiline EPR signals. These signals are, however, very difficult to interpret because the four ${ }^{55} \mathrm{Mn}$ nuclei create more than a thousand EPR lines even for a fixed (unique) orientation of the OEC. The anisotropy of the ${ }^{55} \mathrm{Mn}$ HFI tensors and of the $g$-tensor complicates the powder EPR spectrum of these states even more. To obtain the HFI values of the ${ }^{55} \mathrm{Mn}$ ions, pulse Q-band ${ }^{55} \mathrm{Mn}$-ENDOR was applied to the $S_{2}$ and $S_{0}$ states (Kulik et al. 2005, 2007). The simultaneous simulation of the EPR and ${ }^{55} \mathrm{Mn}-\mathrm{EN}$ DOR spectra yielded reliable principal values for the HFI tensors (Fig. 6). The isotropic HFI values for the case of the exchange-coupled cluster are governed by spin multiplicities of the individual ${ }^{55} \mathrm{Mn}$ ions, which in turn are
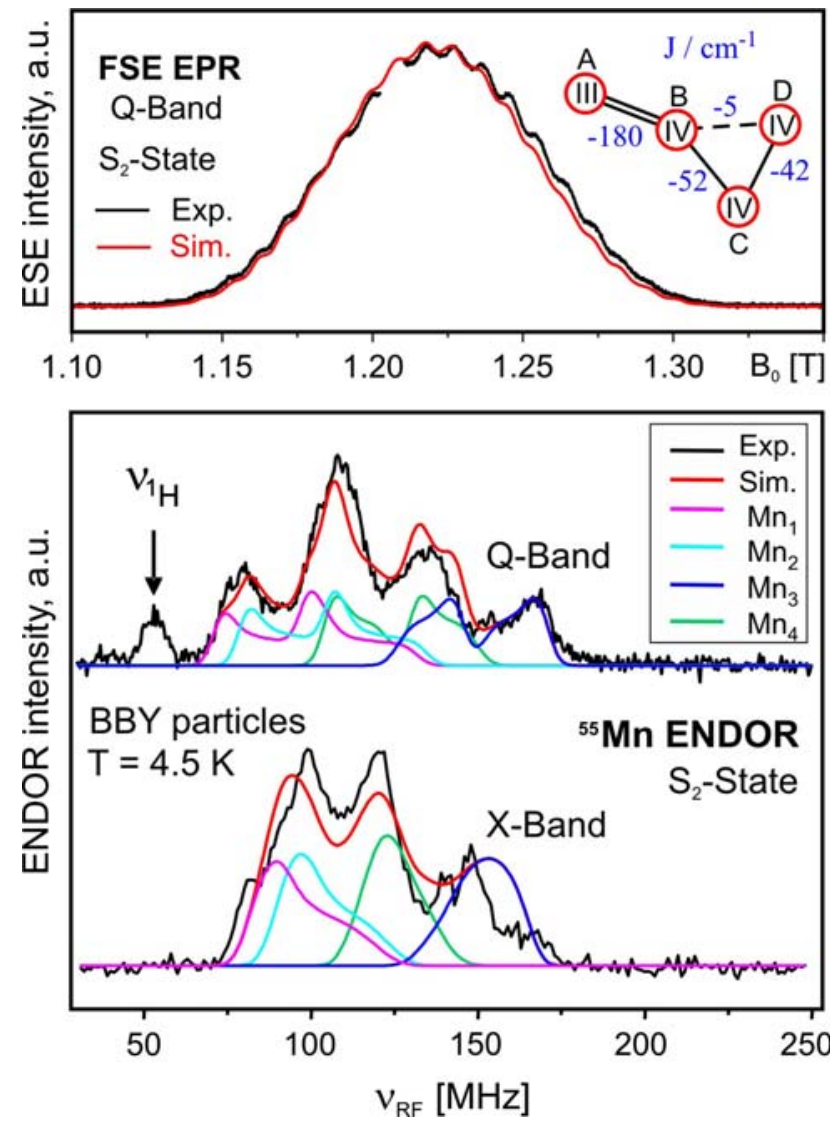

Fig. 6 Top: Field-swept echo detected EPR spectrum at Q-band of the $\mathrm{S}_{2}$-state of the oxygen-evolving complex (OEC) in Photosystem II (BBY particles from spinach). The simulation has been obtained with four axial ${ }^{55} \mathrm{Mn}$ HFI tensors and an anisotropic $g$-tensor (Kulik et al. 2005, 2007). Bottom: ${ }^{55} \mathrm{Mn}$ ENDOR spectra both at Q-band and $\mathrm{X}$-band (black) together with their simulations (red lines) using four different ${ }^{55} \mathrm{Mn}$ hf tensors (colored lines). Note the better nuclear Zeeman resolution at Q-band. The inset in the upper panel shows the assignment of oxidation states to the four Mn ions and the exchange coupling $J$ among these ions

determined by the Mn oxidation states and by the strength of the exchange interactions in the cluster. From the analysis of the ${ }^{55} \mathrm{Mn}$ HFI values, the oxidation state compositions of the OEC could be deduced: $\mathrm{S}_{0} 3 \mathrm{Mn}(\mathrm{III})$ $1 \mathrm{Mn}(\mathrm{IV}) ; \mathrm{S}_{1} 2 \mathrm{Mn}(\mathrm{III}) 2 \mathrm{Mn}(\mathrm{IV}) ; \mathrm{S}_{2} 1 \mathrm{Mn}$ (III) $3 \mathrm{Mn}(\mathrm{IV})$. Furthermore, values for the exchange couplings were obtained and an assignment of the oxidation states to individual Mn ions in the cluster was proposed, see Fig. 6 (Kulik et al. 2007).

\section{Spin-polarized RP $P_{700}^{\bullet+} A_{1}^{\bullet-}$ in plant Photosystem I}

In plant Photosystem I (PSI), the photosynthetic charge separation is triggered by the light absorption of the primary electron donor $P_{700}$. From its excited state $\mathrm{P}^{*}$, the electron is transferred through intermediate acceptors to the electron acceptor $A_{1}$ (vitamin $K_{1}$ ). As a result of the fast 
charge separation, the RP $P_{700}^{\bullet+} A_{1}^{\bullet-}$ is created in a spincorrelated state that can be observed by EPR and ENDOR techniques.

The system of two interacting electron spins has four eigenstates, which can be described in terms of singlet and triplet states. Since spin multiplicity is conserved during fast electron transfer, the system is initially in the singlet state. In the course of spin evolution also the triplet sublevels become populated. The general theory of ESE and ENDOR in polarized RPs is rather complicated (Fursmann et al. 2002; Poluektov et al. 2005). However, the situation is simplified in the weak coupling case, when the difference of the Larmor frequencies of two electron spins $\Delta \omega$ is much larger than the strength of the exchange and magnetic dipolar interactions between these spins. The system approaches this situation with increasing external magnetic field, since $\Delta \omega$ increases due to the difference in $g$-factors of the radicals in the RP.

This was utilized in pulse ENDOR studies of the laser flash generated spin-polarized RP $P_{700}^{\bullet+} A_{1}^{\bullet-}$ (Fursmann et al. 2002; Epel et al. 2006). The Q-band transient EPR spectrum of this RP is shown in the top panel of Fig. 7. The numerical simulation shows that this spectrum is composed of the contributions of the signals of $P_{700}^{\bullet+}$ and $A_{1}^{\bullet-}$, each of which is spin polarized. Further simplification is achieved by measuring the proton ENDOR spectrum at the low-field edge of the EPR spectrum of the RP. At this position, only $A_{1}^{\bullet^{-}}$contributes significantly to the signal intensity. Because of substantial $g$-anisotropy good orientation selection is achieved. The $A_{1}^{\bullet^{-}}$molecules with their molecular $x$-axis oriented along the $B_{0}$ direction give the main contribution to the ESE and ENDOR signals and a single-crystal-like spectrum is obtained in Davies ENDOR experiment (bottom panel of Fig. 7). About 10 line pairs can be distinguished in this ENDOR spectrum, which is nearly symmetrical with respect to the ${ }^{1} \mathrm{H}$ Larmor frequency. Note that this spectrum is very similar to the usual ${ }^{1} \mathrm{H}$ ENDOR spectrum of the chemically generated stationary radical $A_{1}^{\bullet-}$, which supports the assignment of the ENDOR spectrum of the spin-polarized RP $P_{700}^{\bullet+} A_{1}^{\bullet-}$ (Niklas et al. 2009).

The variation of the interpulse delay in the Davies ENDOR pulse sequence leads to a change of the population of the energy levels of the RP. This is reflected in changes of the intensity of the ENDOR lines. In such an experiment, called variable mixing time (VMT) ENDOR (Epel et al. 2006) the ENDOR pattern becomes asymmetric, and some lines even change the sign of the polarization. From this asymmetry, the absolute signs of the HFI constants can be obtained. For $A_{1}^{\bullet-}$, a negative sign of the HFI was derived for the ring $\alpha$-protons and positive signs for methyl and methylene $\beta$-protons, in accordance with theoretical predictions.
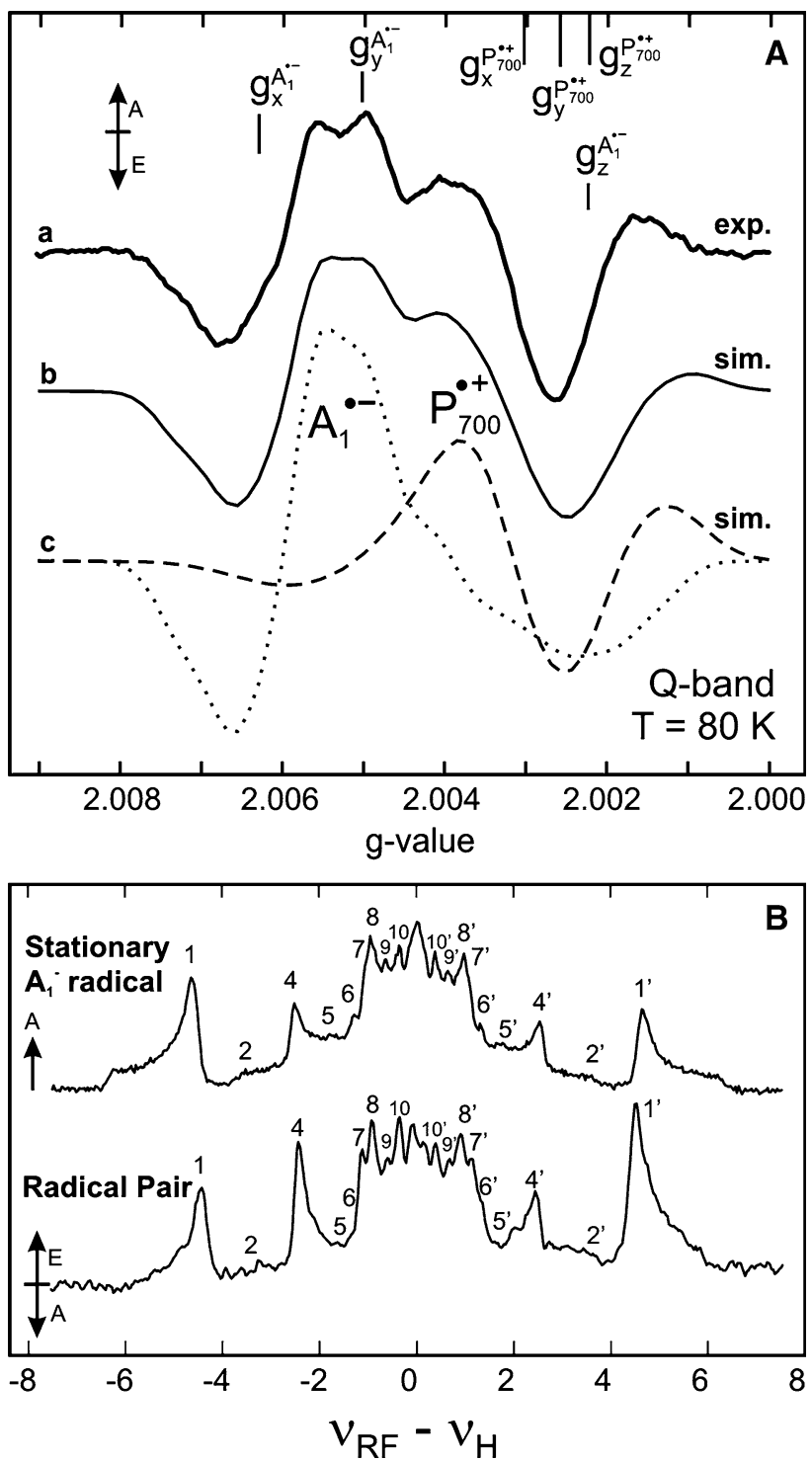

Fig. 7 A: Transient EPR spectrum at Q-band of the in situ lightinduced spin-polarized radical pair (RP) state $P_{700}^{\bullet+} A_{1}^{\bullet-}$ in Photosystem I of Thermosynechococcus elongatus (a) together with its simulation (b); simulations of the individual radicals $\left(P_{700}^{\bullet+}=\mathrm{Chl} a / \mathrm{Chl} a^{\prime}\right.$ dimer; $\mathrm{A}_{1}=$ vitamin $\mathrm{K}_{1}$, electron acceptor) are also shown (c). B: Comparison of ${ }^{1} \mathrm{H}$ ENDOR spectra of the stationary radical $A_{1}^{\bullet-}$ (photo chemical reduction of PSI) and the short-lived RP state $P_{700}^{\bullet+} A_{1}^{\bullet-}$ obtained near $g_{x}\left(A_{1}^{\bullet-}\right)$ where the $P_{700}^{\bullet+}$ contribution is very small. For details see Niklas et al. (2009), Epel et al. (2006)

The carotenoid triplet state in the peridininchlorophyll-protein antenna complex

Photogenerated triplet states can often be observed in bacterial photosynthetic RCs, plant photosystems or the antenna complexes under intense light. In the peridininchlorophyll-protein (PCP) antenna complex from Amphidinium carterae, illumination by red light generates the triplet excited state of the chlorophyll ${ }^{3} \mathrm{Chl} a$. Within a few nanoseconds, the triplet excitation migrates to the 
carotenoid peridinin, which is in optimal contact with the Chl $a \pi$-system. Such quenching of chlorophyll triplets prevents the formation of harmful singlet oxygen and is typical for photosynthetic antenna complexes.

The resulting ${ }^{3} \mathrm{Car}$ state is highly spin polarized. Its EPR spectrum consists of emission and absorption lines, the position of which is determined by the zero-field-splitting (ZFS). Although this state is short-lived, it can be studied by pulse ENDOR if the pulse sequence is completed before the triplet decays to the singlet ground state (Niklas et al. 2007).

Highly resolved Q-band Davies ENDOR spectra were obtained for magnetic field positions corresponding to the canonical orientations of the ZFS tensor (Fig. 8). For the triplet state $(S=1)$, the ENDOR frequencies occur at $v_{\text {ENDOR }}=\left|v_{\mathrm{n}}-M_{s} a\right|$ where $M_{S}= \pm 1,0$. This makes the ENDOR spectrum asymmetric with respect to $v_{\mathrm{n}}$ and allows the direct determination of the signs of the HFI constants relative to the sign of the ZFS parameter $D$. For the studied system, a negative $D$ value was deduced from the analysis of the ENDOR spectra.

Totally nine groups of nonequivalent protons were identified and tentatively assigned to molecular positions based on the comparison of the measured and DFT-calculated HFI tensors. The number of identified protons approximately equals the number of protons in the conjugated part of the peridinin, which confirms that the triplet is localized on one specific peridinin molecule at low temperatures.

\section{Limitations and perspectives of ENDOR spectroscopy}

For CW ENDOR, the major limitation is caused by the need of tuning spin-lattice relaxation rates of electrons and nuclei. For this reason, the CW ENDOR signal usually can be obtained only in a limited temperature range. Besides, at a given temperature the ENDOR lines belonging to some nuclei in a specific sample may disappear, while the lines belonging to other nuclei are still present with good signalto-noise ratio. This may lead to misinterpretations of ENDOR spectra. The problem can partially be solved by using Special TRIPLE spectroscopy. However, the requirement of simultaneous excitation of two nuclear spin transitions poses some limitations. Special TRIPLE can be successfully used only if the frequencies of these transitions are precisely enough determined by the first-order perturbation theory relation, see Eq. 3. Therefore, Special TRIPLE cannot be applied for nuclei with strong HFI. Also it implies the absence of NQI, so Special TRIPLE should not be used for $I>1 / 2$ nuclei in the solid state, unless the NQI is very weak.

The main limitation of pulse ENDOR is the need for relatively long electron spin relaxation times. First, the
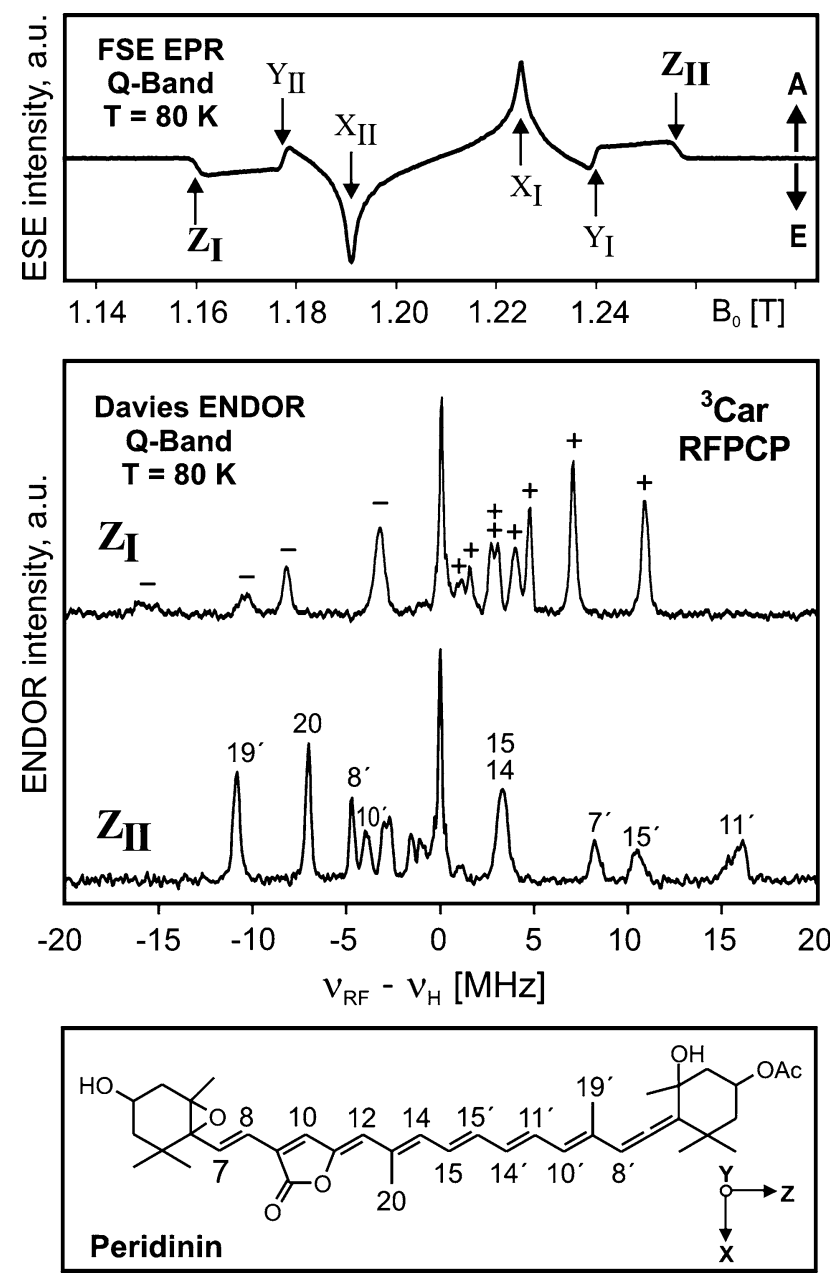

Fig. 8 Top: Field-swept echo EPR at Q-band of the short-lived photoinduced spin-polarized triplet state of the carotenoid peridinin in the PCP (peridinin-chlorophyll-protein) antenna of A. carterae. Middle: Davies ENDOR experiment at Q-band using orientational selection in the EPR with respect to the ZFS tensor axes (positions $\mathrm{Z}_{\mathrm{I}}$ and $\mathrm{Z}_{\mathrm{II}}$ ). Note that lines with positive HFI constants appear on the high (low) frequency side of the spectrum and with negative signs on the low (high) frequency side, for the EPR field position $Z_{I}\left(Z_{I I}\right)$. Thus, magnitude and signs of the couplings are directly available from the spectrum. For the peridinin triplet, at least $12{ }^{1} \mathrm{H}$ HFI constants were obtained. From the assigned couplings, the spin density distribution in the molecule can be constructed and compared with that obtained from DFT calculations. Bottom: Molecular structure of peridinin including axis system: For details see Niklas et al. (2007)

transverse relaxation time $T_{2}$ should be long enough to obtain an ESE signal with sufficient intensity. This is not always the case, for example, no ESE signal is still obtained for the artificially reduced $\mathrm{S}_{-2}$ state of the OEC in PSII and for the $Q_{A}^{\bullet-} \mathrm{Fe}^{2+}$ complex in the bacterial RC, despite the pronounced CW EPR signals recorded for these systems. Second, $T_{1}$ should be long enough to allow the application of the rf pulse before the non-equilibrium electron magnetization created by the preparation $\mathrm{mw}$ pulse relaxes. This often demands deep cooling of the 
sample, e.g., for the case of transition metal complexes like the Mn-cluster (OEC) in PSII. Under such conditions "heating artifacts" may appear in the ENDOR spectra. Their origin is the heat which is released in the rf coils during the rf pulse. This heat is experienced by the cavity and also by the sample where it increases $T_{1}$. This, in turn, causes a variation of the degree of ESE inversion by the preparation pulse. If the heat release depends on the rf, a distortion of the ENDOR spectrum will result. The most effective way of avoiding such distortions is random $\mathrm{rf}$ sampling during the acquisition of the ENDOR spectrum ("stochastic ENDOR"), which suppresses the rf-induced heat accumulation (Epel et al. 2003).

In Davies ENDOR, the signal intensity is decreased when both EPR transitions (different $m_{I}$ ) of a particular nucleus are excited by the preparation mw pulse. For this reason, Davies ENDOR does not work well for nuclei with small HFI constants. This is not a severe limitation for protons, because the proton gyromagnetic ratio is large and the HFI with protons is typically strong. However, this becomes important for nuclei with small gyromagnetic ratio $\left({ }^{2} \mathrm{H},{ }^{17} \mathrm{O}\right.$, and others), which often have quite small HFI constants. In this case, Mims ENDOR can be applied. However, Mims ENDOR suffers from blindspots in the spectrum, so ESEEM techniques are sometimes the better choice for the detection of nuclei with small HFI.

Although not discussed in the present paper, high-field/ high-frequency ENDOR is very interesting for photosynthetic studies (Möbius and Savitsky 2008). First, with increasing mw frequency the EPR signal intensity grows, while the necessary sample volume is decreased. This is especially important for costly preparations, such as single crystals or genetically modified systems. Second, the spectral resolution increases in both EPR and NMR dimensions. The latter is caused by the increasing nuclear Larmor frequency. The ENDOR lines from different nuclei, which overlap at conventional X-band, become separated at high field.

The pulse ENDOR study of short-lived paramagnetic intermediates, such as spin-correlated RPs and triplet states in the photosystems, is highly important for understanding the primary steps of photosynthesis. In RPs, the unusual out-of-phase ESE signal appears which can be used for pulse ENDOR detection. Although several ENDOR investigations of photosynthetic spin-correlated RPs have been reported, the lack of a simple theory of such systems complicates the interpretation of the results.

Acknowledgments The authors thank the coworkers named in the references for their important contribution to this work. Financial support was obtained from the Max Planck Society and the DFG ( $\mathrm{Sfb}$ 663, TP A7), and from Russian Foundation for Basic Research (6-0448021a). The President of Russian Federation grant for scientific schools (HII-551.2008.3) is also acknowledged.

\section{References}

Biehl R, Plato M, Möbius K (1975) General TRIPLE resonance on free radicals in solution. Determination of relative signs of isotropic hyperfine coupling constants. J Chem Phys 63:35153522. doi: $10.1063 / 1.431790$

Britt RD, Campbell KA, Peloquin JM, Gilchrist ML, Aznar CP, Dicus MM, Robblee J, Messinger J (2004) Recent pulsed EPR studies of the Photosystem II oxygen-evolving complex: implications as to water oxidation mechanisms. Biochim Biophys Acta 1655:158-171. doi:10.1016/j.bbabio.2003.11.009

Davies ER (1974) A new pulse ENDOR technique. Phys Lett A 47: 1-2. doi:10.1016/0375-9601(74)90078-4

Dinse KP, Biehl R, Möbius K (1974) Electron nuclear triple resonance of free radicals in solution. J Chem Phys 61:43354341. doi:10.1063/1.1681740

Epel B, Arieli D, Baute D, Goldfarb D (2003) Improving W-band pulsed ENDOR sensitivity-random acquisition and pulsed special TRIPLE. J Magn Reson 164:78-83. doi:10.1016/S10907807(03)00191-5

Epel B, Niklas J, Antonkine ML, Lubitz W (2006) Absolute signs of hyperfine coupling constants as determined by pulse ENDOR of polarized radical pairs. Appl Magn Reson 30:311-327

Feher G (1956) Observation of nuclear magnetic resonances via the electron spin resonance line. Phys Rev 103:834-835. doi: 10.1103/PhysRev.103.834

Flores M, Isaacson R, Abresch E, Calvo R, Lubitz W, Feher G (2007) Protein-cofactor interaction in bacterial reaction centers from Rhodobacter sphaeroides R-26: geometry of the hydrogen bonds to the primary quinone $Q_{A}^{\bullet-}$ by ${ }^{1} \mathrm{H}$ and ${ }^{2} \mathrm{H}$ ENDOR spectroscopy. Biophys J 82:671-682

Freed JH (1969) Theory of saturation and double resonance effects in ESR spectra. IV. Electron-nuclear triple resonance. J Chem Phys 50:2271-2272. doi:10.1063/1.1671371

Fursmann CE, Teutloff C, Bittl R (2002) Pulsed ENDOR studies of shortlived spin-correlated radical pairs in photosynthetic reaction centers. J Phys Chem B 106:9679-9686. doi:10.1021/jp0257202

Hyde JS, Maki AH (1964) ENDOR of a free radical in solution. J Chem Phys 40:3117-3118. doi:10.1063/1.1724957

Kevan L, Kispert L (1976) Electron spin double resonance spectroscopy. Wiley Interscience, New York, USA

Kulik LV, Epel B, Lubitz W, Messinger J (2005) ${ }^{55} \mathrm{Mn}$ pulse ENDOR

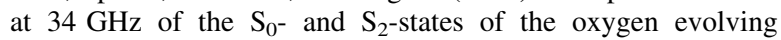
complex in Photosystem II. J Am Chem Soc 127:2392-2393. doi:10.1021/ja043012j

Kulik LV, Epel B, Lubitz W, Messinger J (2007) Electronic structure of the $\mathrm{Mn}_{4} \mathrm{O}_{\mathrm{x}} \mathrm{Ca}$ cluster in the $\mathrm{S}_{0}$ and $\mathrm{S}_{2}$ states of the oxygenevolving complex of Photosystem II based on pulse ${ }^{55} \mathrm{Mn}$ ENDOR and EPR spectroscopy. J Am Chem Soc 129:1342113425. doi:10.1021/ja071487f

Kurreck K, Kirste B, Lubitz W (1988) Electron-nuclear double resonance spectroscopy of radicals in solution: application to organic and biological chemistry. $\mathrm{VCH}$, New York

Lendzian F, Huber M, Isaacson RA, Endeward B, Plato M, Bönigk B, Möbius K, Lubitz W, Feher G (1993) Electronic structure of the primary donor cation radical in Rhodobacter sphaeroides R-26: ENDOR and TRIPLE resonance studies in single crystals of reaction centers. Biochim Biophys Acta 1183:139-160. doi: 10.1016/0005-2728(93)90013-6

Lubitz W, Feher G (1999) The primary and secondary acceptors in bacterial photosynthesis III. Characterization of the quinone radicals $Q_{A}^{\bullet-}$ and $Q_{B}^{\bullet-}$ by EPR and ENDOR. Appl Magn Reson $17: 1-48$

Lubitz W, Lendzian F (1996) ENDOR spectroscopy. In: Amesz J, Hoff AJ (eds) Biophysical techniques in photosynthesis. 
Advances in photosynthesis and respiration, vol 3. Kluwer Academic Publisher, Dordrecht, pp 255-275

Lubitz W, Lendzian F, Plato M, Scheer H, Möbius K (1997) The bacteriochlorophyll $a$ cation radical revisited. An ENDOR and TRIPLE resonance study. Appl Magn Reson 13:531-551

Lubitz W, Lendzian F, Bittl R (2002) Radicals, radical pairs and triplet states in photosynthesis. Acc Chem Res 35:313-320. doi: 10.1021/ar000084g

Mims WB (1965) Pulsed ENDOR experiments. Proc R Soc Lond A 283:452-457

Möbius K, Savitsky A (2008) High-field EPR spectroscopy on proteins and their model systems. Characterization of transient paramagnetic states. RSC Publishing, World Society of Chemistry, Cambridge, UK

Möbius K, Lubitz W, Plato M (1989) Liquid state ENDOR and TRIPLE resonance. In: Hoff AJ (ed) Advanced EPR: applications to biology and biochemistry. Elsevier, The Netherlands, pp 441-499

Niklas J, Schulte T, Prakash S, van Gastel M, Hofmann E, Lubitz W (2007) Spin-density distribution of the carotenoid triplet state in the peridinin-chlorophyll-protein antenna. A Q-band pulse electron-nuclear double resonance and density functional theory study. J Am Chem Soc 129:15442-15443. doi:10.1021/ja $077225 \mathrm{v}$

Niklas J, Epel B, Antonkine ML, Sinnecker S, Pandelia ME, Lubitz W (2009) The electronic structure of the secondary electron acceptor $A_{1}^{\bullet-}$ of photosystem I investigated by advanced pulse EPR and ENDOR techniques. J Phys Chem B (submitted)
Plato M, Lubitz W, Möbius K (1981) A solution ENDOR sensitivity study of various nuclei in organic radicals. J Phys Chem 85:1202-1219. doi: $10.1021 / \mathrm{j} 150609 \mathrm{a} 024$

Poluektov OG, Utschig LM, Dubinskij AA, Thurnauer MC (2005) Electron transfer pathways and protein response to charge separation in photosynthetic reaction centers: time-resolved high-field ENDOR of the spin-correlated radical pair $P_{865}^{\bullet+} Q_{A}^{\bullet+}$. J Am Chem Soc 127:4049-4059. doi:10.1021/ja043063g

Poole CP Jr (1983) Electron spin resonance. A comprehensive treatise on experimental techniques. Wiley Intersience, New York, USA

Rigby SEJ, Evans MCW, Heathcote P (2001) Electron nuclear double resonance (ENDOR) spectroscopy of radicals in photosystem I and related Type 1 photosynthetic reaction centres. Biochim Biophys Acta 1507:247-259. doi:10.1016/S0005-2728(01) 00211-0

Schweiger A, Jeschke G (2001) Principles of pulse electron paramagnetic resonance. Oxford University Press, UK

Sinnecker S, Koch W, Lubitz W (2000) Bacteriochlorophyll $a$ radical cation and anion-calculation of isotropic hyperfine coupling constants by density functional methods. Phys Chem Chem Phys 2:4772-4778. doi:10.1039/b004370m

Sinnecker S, Flores M, Lubitz M (2006) Protein-cofactor interactions in bacterial reaction centers from Rhodobacter sphaeroides $\mathrm{R}$ 26: effect of hydrogen bonding on the electronic and geometric structure of the primary quinone. A density functional theory study. Phys Chem Chem Phys 8:5659-5670. doi:10.1039/ b612568a 